\title{
Correlation between Photoluminescence Properties of Surface Defects and Laser-Induced Damage Threshold of Fused Silica
}

\author{
Qiao Chen, ${ }^{1}$ He Wang, ${ }^{1}$ Rucheng Dai, ${ }^{2}$ Zhongping Wang, ${ }^{2}$ Xiaoping Tao, ${ }^{2}$ Wei Zhao $\mathbb{D},{ }^{2}$ \\ and Zengming Zhang $\mathbb{D}^{1,2}$ \\ ${ }^{1}$ Department of Physics and CAS Key Laboratory of Strong-Coupled Quantum Matter Physics, \\ University of Science and Technology of China, Hefei, Anhui 230026, China \\ ${ }^{2}$ The Center of Physical Experiments, University of Science and Technology of China, Hefei 230026, China
}

Correspondence should be addressed to Wei Zhao; weiz3@ustc.edu.cn and Zengming Zhang; zzm@ustc.edu.cn

Received 2 February 2021; Accepted 11 June 2021; Published 23 June 2021

Academic Editor: Dieter H.H. Hoffmann

Copyright (c) 2021 Qiao Chen et al. This is an open access article distributed under the Creative Commons Attribution License, which permits unrestricted use, distribution, and reproduction in any medium, provided the original work is properly cited.

Laser-induced damage threshold is the main limitation for fused silica optics in high-power laser applications. The existence of various defects near the surface is the key factor for the degradation of the threshold. In this work, the photoluminescence spectra at different regions of the damaged and recovered fused silica samples are recorded to analyze the correlation between photoluminescence of surface defects and laser-induced damage threshold. The experimental data concluded the inverse proportional correlation between fluorescence and laser-induced damage threshold value. The weak photoluminescence is the guarantee of the high laser-induced damage threshold, and then the higher local Si nanocluster concentration corresponds with the higher laserinduced damage threshold value for the fused silica optics after $\mathrm{CO}_{2}$ laser treatment. The investigation reveals that photoluminescence measurement can be employed to check the quality of pristine fused silica and evaluate the tendency of the laserinduced damage threshold value. The current results are helpful for understanding the evolution of interaction from $\mathrm{CO}_{2}$ laser treatment and fused silica optics and can provide the guide of process technology for the high quality of fused silica optics.

\section{Introduction}

Fused silica optics is widely used in high-power laser facilities such as inertial confinement fusion (ICF) due to its high transparency and excellent thermal and chemical stabilities. However, there exist some intrinsic defects and many kinds of flaws on the surface associated with the fabrication process of fused silica optics. These defects could absorb ultraviolet laser and cause the initial laser damage [1] and then further degrade the laser-induced damage threshold (LIDT) of fused silica optics. Some approaches, such as ion beam etching $[2,3]$, chemical etching [1, 4], and $\mathrm{CO}_{2}$ laser treatment $[5,6]$, have been employed to mitigate the initial damage sites. Among these methods, $\mathrm{CO}_{2}$ laser treatment is one important method to repair the initial damage site by heating effect. Some investigations demonstrated that the LIDT may return to the normal level or higher after $\mathrm{CO}_{2}$ laser treatment $[7,8]$. Although there have been some reports on the optical and mechanical effects of $\mathrm{CO}_{2}$ laser treatment for scratches and laser-induced damage sites on the surface of fused silica, as well as the physical model of the secondary treatment or annealing process to eliminate residual stress $[9,10]$, there is no report about the evolution of defects on the fused silica surface during this process.

Some works revealed that the intrinsic defects and laserinduced structural defects in fused silica optics include the $E^{\prime}$-center [11], which comprised an unpaired electron of single silicon bonded to just three oxygens, oxygen-deficiency center (ODC) [12], nonbridging oxygen hole center (NBOHC) [13], peroxy radical (POR) [14], and Si nanocluster [15]. The presence of defects in the silica matrix can dramatically change its structural, electrical, and optical properties. So, photoluminescence (PL) spectra are one significant tool to understand the evolution of the defects in the fused silica optics during high-power laser irradiation. 
According to PL analysis, the electron structural information of various defect species and the distribution of defects on the surface should be obtained for fused silica optics.

Some groups investigated the luminescence properties of the defects in fused silica in the past few decades. Demos et al. assigned the luminescent bands around $400 \mathrm{~nm}$ and $650 \mathrm{~nm}$ to ODC and NBOHC defects [16], separately. The other emission bands in the range from $450 \mathrm{~nm}$ to $600 \mathrm{~nm}$ are attributed to the combination of the self-trapped exciton (STE). Švrček et al. concluded that the emission band of the $\mathrm{Si}$ nanocluster can be in a wide range around $700 \mathrm{~nm}$. The concentration of various defects in the laser-induced initial damage site would increase dramatically compared with the pristine area. The initial damage site will probably expand exponentially due to higher defect density as reported [17-19].

In this paper, PL properties of defects near the surface for the laser damage site and $\mathrm{CO}_{2}$ laser treatment site are systematically investigated. The correlation between the distribution of different defects and LIDT in the same site is analyzed.

\section{Experimental}

The experiments were conducted on UV-grade Corning 7980 fused silica samples with $40 \mathrm{~mm}$ square and a thickness of $4 \mathrm{~mm}$. They were firstly etched in the buffered hydrofluoric (HF) acid solutions and then cleaned by deionized water.

Laser-induced damage sites were generated on the output surface of pristine samples by using a triple-frequency Nd:YAG laser (Saga) which delivers a pulse width of $6.3 \mathrm{~ns}$ at $355 \mathrm{~nm}$ with a diameter of $800 \mu \mathrm{m}$ at $1 / e^{2}$. The diameter of the damage area is about $250 \mu \mathrm{m}$.

The $\mathrm{CO}_{2}$ laser of the repairing treatment is a commercial radio frequency power excited $\mathrm{CO}_{2}$ laser (Coherent GEM$100 \mathrm{~L}$ ), with a maximum output power of 100 watt and power stability of $\pm 3 \%$.

The photoluminescence spectra were recorded by using a spectrometer (LabRAM HR800, Jobin Yvon) with a confocal microscope and a multichannel air-cooled CCD detector. The excitation sources were an Ar ion laser $\left(\lambda_{0}=514.5 \mathrm{~nm}\right.$; source power: $20 \mathrm{~mW})$ and He-Cd laser $\left(\lambda_{0}=325 \mathrm{~nm}\right.$; source power: $40 \mathrm{~mW})$. All spectra were recorded at room temperature.

Laser-induced damage threshold is obtained by the R-on-1 testing mode. The schematic diagram of the measurement setup is shown in Figure 1. On each selected site, the laser fluence was progressively increasing, until the first damage occurred. For each set of parameters, a series of sites were selected and analyzed, and the results given in this work are based on a statistical analysis on the fused silica samples.

\section{Results and Discussion}

Figure 2(a) shows the photoluminescence spectra at the pristine region and the damaged region of the fused silica sample with the excitation of $325 \mathrm{~nm}$ laser. The corresponding decomposition peaks are displayed in Figures 2(b) and 2(c), respectively. The shoulder peak around $400 \mathrm{~nm}$ is from ODC defects. The broad emission bands centered at $490 \mathrm{~nm}$ and $580 \mathrm{~nm}$ can be definitely ascribed to the transition from the laser-induced STE $[12,13]$. The peaks around $650 \mathrm{~nm}$ and $750 \mathrm{~nm}$ are assigned to the luminescence bands of $\mathrm{NBOHC}$ and $\mathrm{Si}$ nanocluster defects, respectively. The weak emission indicates the low concentration for these defects in the clear pristine sample. These defects in pristine fused silica optics can have a high absorption for the UV laser and become an initial laser damage site according to existing reports $[1,11,12]$. In other words, the low defects' density can guarantee the high LIDT value. The contribution from the NBOHC and Si nanocluster is hardly observable for the pristine region, but the obvious peaks occur for the two defects in the damaged region as seen in Figure 2.

Figure 3 compares the photoluminescence spectra of the pristine region, ion beam etching-treated region, and the edge region of the laser-induced damage site with the excitation of a $514.5 \mathrm{~nm}$ laser. The sharp peaks at $526 \mathrm{~nm}$, $536 \mathrm{~nm}$, and $543 \mathrm{~nm}$ are the response to Raman modes for $\mathrm{Si}-\mathrm{O}-\mathrm{Si}$, fourfold rings, and threefold rings in fused silica, respectively [11].

The broad weak fluorescence of curve \#2 in Figure 3 for the pristine region is similar to that in Figure 2 with $325 \mathrm{~nm}$ excitation. Ion beam etching process can smooth the surface of the optics and mitigate a part of the existing surface defects. The microscopic image of the etching region is shown in Figure 3(c). It is smoother and has higher uniformity compared with the pristine region.

Except for Raman peaks, no emission bands can be detected for the ion beam etching-treated sites as seen in curve \#3 of Figure 3(a). This verified that the ion beam etching process can remove the flaws localized on the surface and enhance the LIDT value.

The strong luminescent band centered at $580 \mathrm{~nm}$ is quite prominent as shown in curve \#1 of Figure 3(a) for the edge region of the laser-induced damage area. It indicates that the laser-induced damage process generates a higher concentration of defects. In general, the type and relative concentration of defects depend on the peak position and intensity of PL spectra, respectively. As the description given above, the laser-induced self-trapped excitons dominate the emission around $580 \mathrm{~nm}$. These defects could result in the damage of fused silica under ultrahigh power running of the UV laser due to the strong absorption. Investigating the influence on the LIDT from these defects, the values of LIDT at different sites are measured by the R-on-1 testing mode [20]. The LIDT, the type, and the relative concentration of defects for different regions are given in Table 1. The LIDT values for the pristine surface and ion beamtreated region are $13.9 \mathrm{~J} / \mathrm{cm}^{2}$ and $17.2 \mathrm{~J} / \mathrm{cm}^{2}$, separately. The low LIDT value of $4 \mathrm{~J} / \mathrm{cm}^{2}$ is the response to the edge region of the laser-induced damage site. The results from Table 1 reveal that the PL intensity is inversely proportional to the LIDT value of the fused silica optics. Further comparing the contribution from different defects for the pristine region and damaged region, the laser-induced STEs 


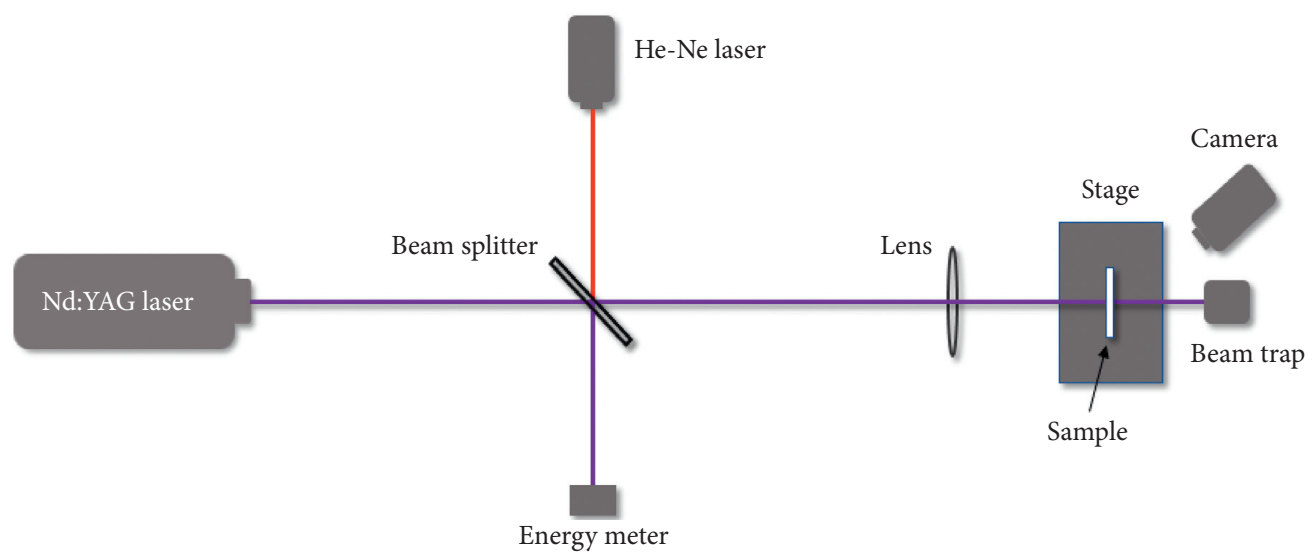

FIGURE 1: Schematic diagram of the laser-induced damage threshold measurement. The setup consists of a single-longitudinal-mode Nd: YAG laser at $355 \mathrm{~nm}$, a He-Ne laser collimating light source, a beam splitter, a focusing lens, an energy meter, and a two-dimensional stage.

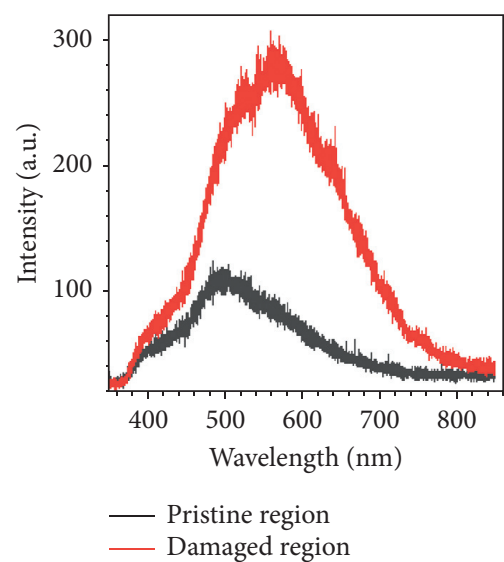

(a)
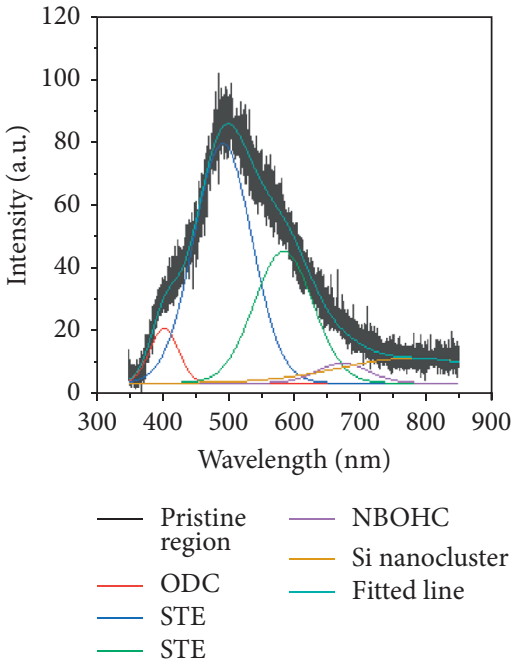

(b)
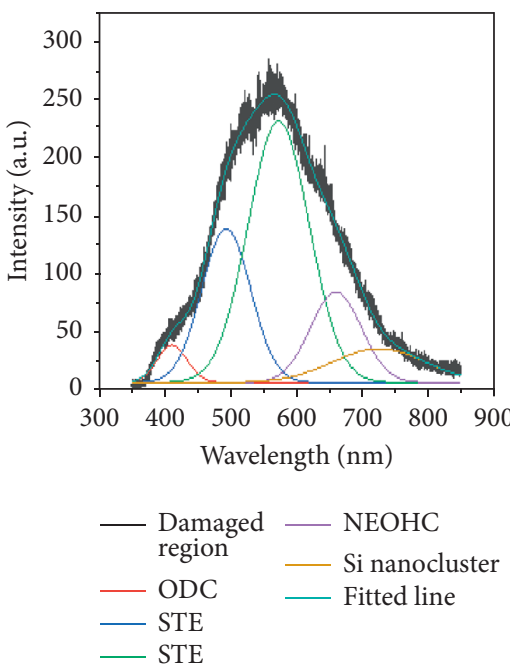

(c)

Figure 2: (a) PL spectra of the pristine region and damaged region of the fused silica sample with $325 \mathrm{~nm}$ excitation. The decomposition peaks for (b) the pristine region and (c) the damaged region.

dominate the damaged threshold value. These results indicate that the PL can be employed to check the quality of pristine fused silica and evaluate the possible LIDT value based on the confirmed reference standard sample.

$\mathrm{CO}_{2}$ laser treatment is the most effective method for fused silica materials to mitigate the defects on the surface. Figure 4(a) shows the optical microscopy image of the $\mathrm{CO}_{2}$ laser treatment site. The PL spectra of three different regions are shown in Figure 4(b). This laser-induced damage site has been overtreated by the $\mathrm{CO}_{2}$ laser, and the ablation trace can be observed in its edge region. The PL spectrum for the edge region has relative high intensity around $500 \mathrm{~nm}$ from the laser-induced STE and the shoulder at $400 \mathrm{~nm}$ from the ODC according to the previous reports $[13,14]$. The measured LIDT value in this region is $4.5 \mathrm{~J} / \mathrm{cm}^{2}$, and it supports the same inverse proportional correlation between PL band intensity centered at $500 \mathrm{~nm}$ and the LIDT.

In spite of the weak fluorescence for center regions 1 and 2, a new luminescence band centered at $750 \mathrm{~nm}$ obviously occurs. This band is ascribed to the $\mathrm{Si}$ nanoclusters. The fabrication process and $\mathrm{Si}$-implanted $\mathrm{a}-\mathrm{SiO}_{2}$ after $1100^{\circ} \mathrm{C}$ annealing can generate the nanoclusters [21]. These inducing paths are all related to the $\mathrm{CO}_{2}$ laser heating process. The intensity at center region 1 is higher due to near the center and bathing in higher temperature during the laser irradiation. These results are quite helpful to understand the mechanism of defects' evolution of the $\mathrm{CO}_{2}$ laser treatment process. It should also be noticed that the LIDT value in center region 1 of the $\mathrm{CO}_{2}$ laser-treated site is $19.2 \mathrm{~J} / \mathrm{cm}^{2}$ higher than $17 \mathrm{~J} / \mathrm{cm}^{2}$ for center region 2 as shown in Figure 5. This indicates that $\mathrm{CO}_{2}$ laser-treated fused silica optics can run under higher fluence compared to the pristine fused silica optics.

Combining Table 1 with Figures 4 and 5, we can conclude that the weak photoluminescence can be taken as the criterion of the high LIDT value; furthermore, the higher nanocluster contribution protects the damage of fused silica under higher laser power running. In fused silica materials, 


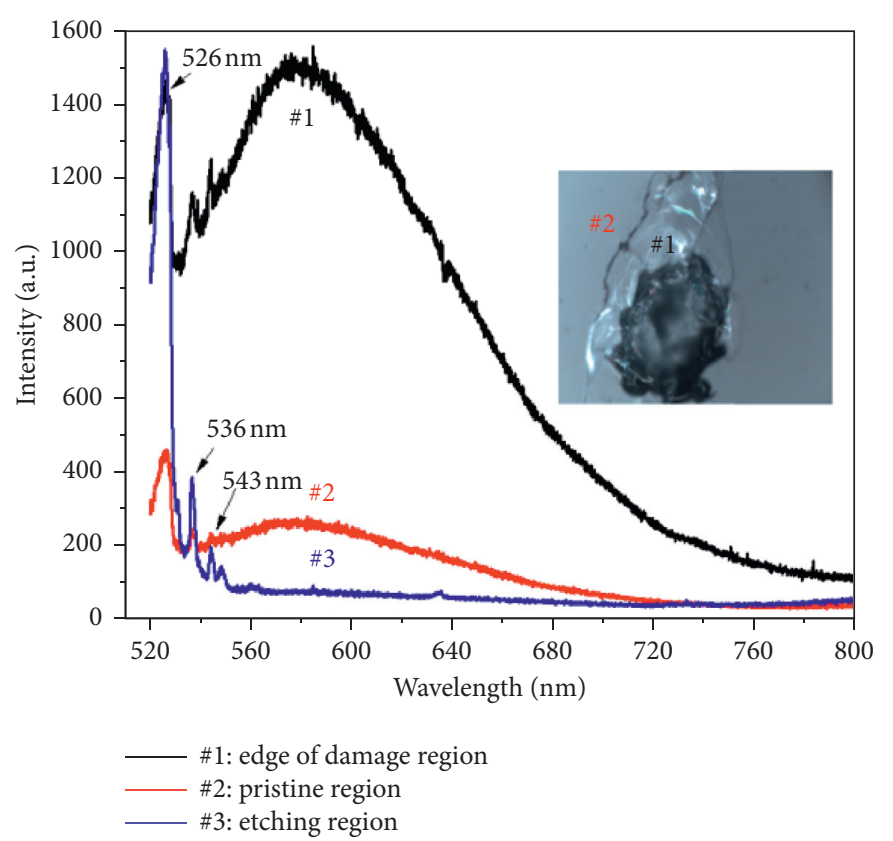

(a)

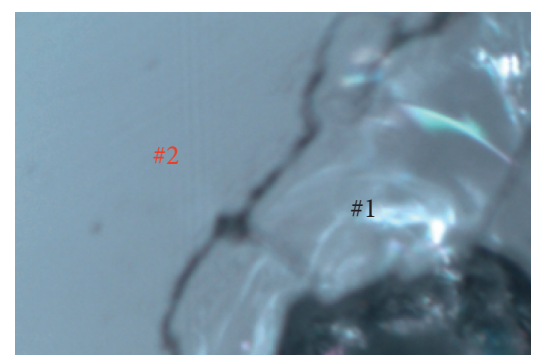

(b)

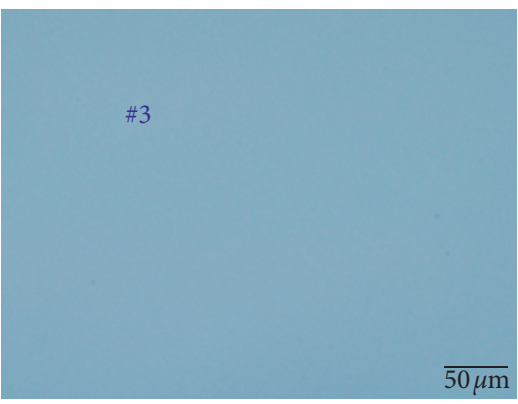

(c)

Figure 3: (a) PL spectra of the fused silica sample with $514.5 \mathrm{~nm}$ excitation. Curve \#1: pristine region, curve \#2: the edge region of the laserinduced damaged site, and curve \#3: the ion beam etching-treated region. The inset is the microscopic image of the selected damage site. (b) The magnification of the inset. (c) The microscopic image of the etching region.

TABLE 1: LIDT and relative fluorescence intensity of several types of defects in different regions.

\begin{tabular}{|c|c|c|c|}
\hline Measured region & Pristine region & Ion beam-treated region & Edge region of the damaged site \\
\hline LIDT & $13.9 \mathrm{~J} / \mathrm{cm}^{2}$ & $17.2 \mathrm{~J} / \mathrm{cm}^{2}$ & $4.0 \mathrm{~J} / \mathrm{cm}^{2}$ \\
\hline ODC & $5.8(2.3)$ & Undetectable & 3.5 \\
\hline STE & $80.4(32.2)$ & Undetectable & 72.7 \\
\hline $\mathrm{NBOHC}$ & $3.8(1.5)$ & Undetectable & 14.4 \\
\hline Si nanoclusters & $10.0(4.0)$ & Undetectable & 9.4 \\
\hline
\end{tabular}

The PL intensity is normalized. The intensity of the pristine region possesses $40 \%$ of the intensity for the damaged site. The value in the bracket for the pristine region is the relative ratio to the PL intensity of the damaged region. 


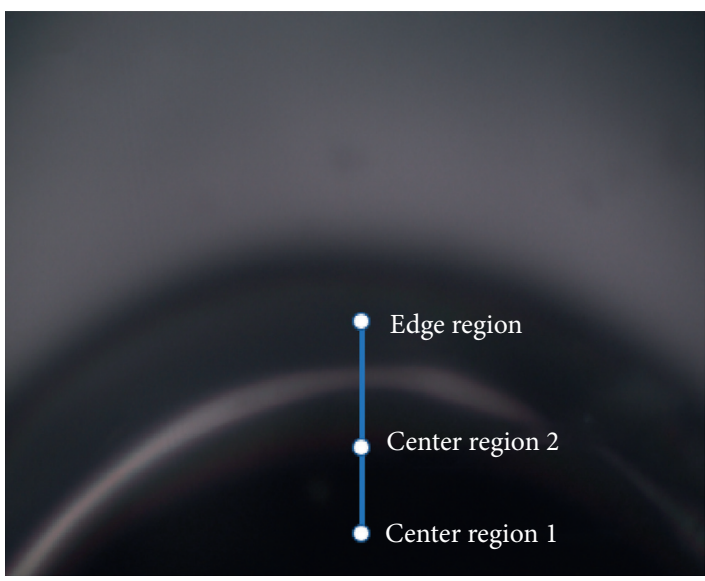

(a)

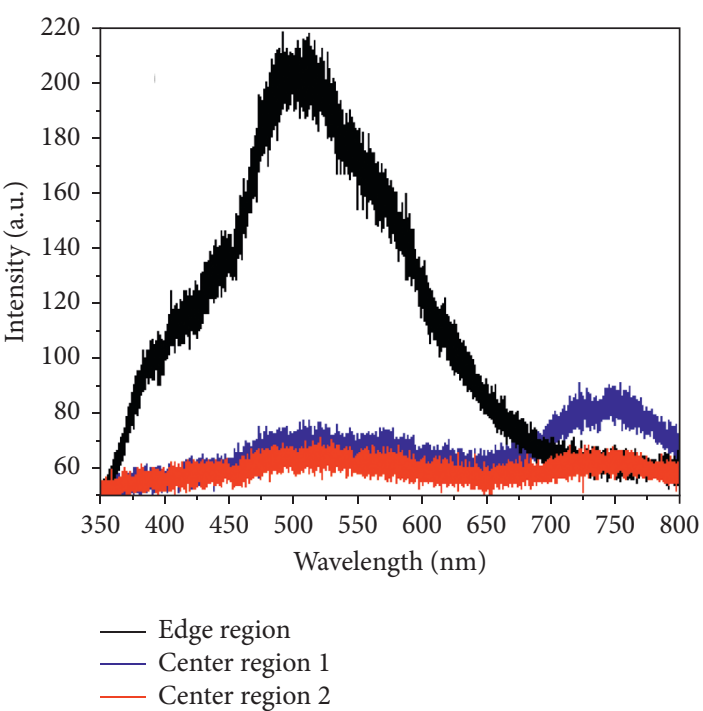

(b)

Figure 4: (a) Optical microscopy images of the $\mathrm{CO}_{2}$ laser treatment site. (b) PL spectra of different regions under $325 \mathrm{~nm}$ excitation.

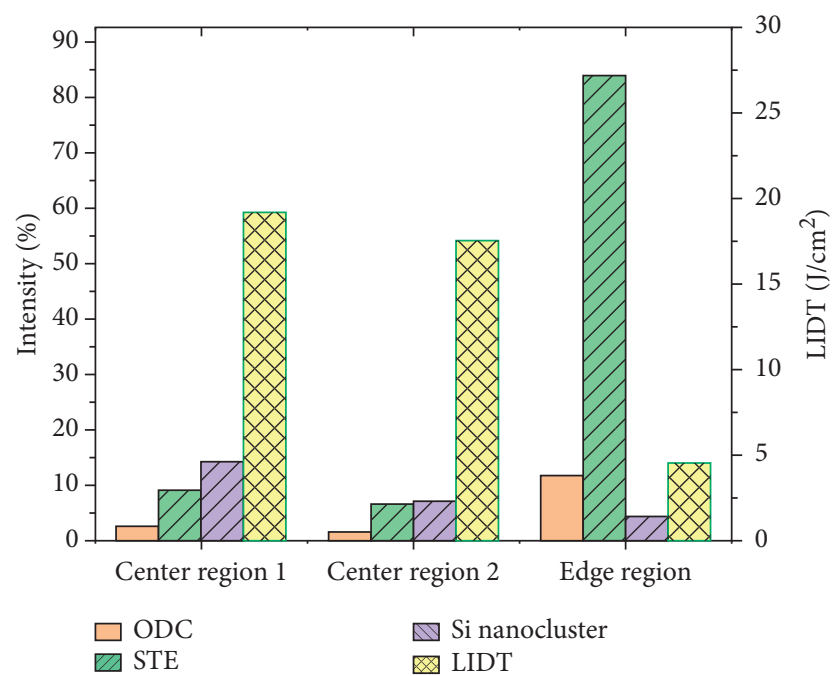

FIGURE 5: Luminescent intensities of defects and LIDT values for different regions in Figure 4. Each photoluminescence spectrum area is normalized to that at the edge region.

the self-trapped exciton is known to be correlated with the absorption precursor according to the existing reports; this is consistent with the results shown in Figure 5. The relationship between the LIDT and Si nanocluster needs to be studied further.

\section{Conclusions}

PL technique combined with the laser-induced damage threshold measurement is employed to investigate the distribution of the defects on the surface of the fused silica sample. The inverse proportional correlation between fluorescence and LIDT value is built according to the experimental results. The weak photoluminescence and the higher Si nanocluster concentration can enhance the LIDT value for the fused silica optics after $\mathrm{CO}_{2}$ laser treatment. The investigation reveals that the fluorescence can be employed to check the quality of pristine fused silica and evaluate the possible laser-induced damage threshold value. The wide emission from the ODC and self-trapped excitons dominates the low LIDT value. The current results are helpful for understanding the evolution of action from $\mathrm{CO}_{2}$ laser treatment and fused silica optics.

\section{Data Availability}

The data used to support the findings of this study are available from the corresponding author upon request.

\section{Disclosure}

Qiao Chen and He Wang are the coauthors.

\section{Conflicts of Interest}

The authors declare that they have no conflicts of interest.

\section{Acknowledgments}

This work was supported by the National Natural Science Foundation of China (nos. 12074360, 21905263, and 51677175), the Science Challenge Project (no. TZ2016001), and the Fundamental Research Funds for the Central Universities.

\section{References}

[1] P. Miller, T. Suratwala, J. Bude et al., Laser Damage Precursors In Fused Silica, Lawrence Livermore National Laboratory, Livermore, CA, USA, 2009. 
[2] T. Kamimura, S. Akamastsu, M. Yamamoto et al., "Enhancement of surface-damage resistance by removing subsurface damage in fused silica," in Proceedings of the Annual Symposium on Optical Materials for High Power Lasers: Boulder Damage Symposium, pp. 244-249, Boulder, CO, USA, June 2004.

[3] I. Adesida, A. T. Ping, C. Youtsey et al., "Characteristics of chemically assisted ion beam etching of gallium nitride," Applied Physics Letters, vol. 65, no. 7, pp. 889-891, 1994.

[4] T. Tankahagi, A. Ishitani, H. Kuroda et al., "Fluorine-containing species on the hydrofluoric acid etched silicon singlecrystal surface," Journal of Applied Physics, vol. 69, no. 2, pp. 803-807, 1911.

[5] J. J. Adams, M. Bolourchi, J. D. Bude et al., Results Of Applying A Non-Evaporative Mitigation Technique To Laser-Initiated Surface Damage On Fused-Silica, International Society for Optics and Photonics Bellingham, WA, USA, 2010.

[6] M. D. Feit and A. M. Rubenchik, Mechanisms Of $\mathrm{CO}_{2}$ Laser Mitigation Of Laser Damage Growth In Fused Silica, pp. 91-102, International Society for Optics and Photonics Bellingham, WA, USA, 2003.

[7] R. M. Brusasco, B. M. Penetrante, J. A. Butler, S. M. Maricle, and J. E. Peterson, " $\mathrm{CO}_{2}$-Laser polishing for reduction of 351nm surface damage initiation in fused silica," in Proceedings of the Annual Symposium on Optical Materials for High Power Lasers, pp. 1-2, Boulder, CO, USA, January 2001.

[8] R. Brusasco, B. M. Penetrante, J. A. Butler et al., "Localized $\mathrm{CO}_{2}$ laser treatment for mitigation of $3 \omega$ damage growth on fused silica," pp. 1-3, Boulder Damage Symposium on Optical Material, Boulder, CO, USA, 2001.

[9] T. Doualle, L. Gallais, P. Cormont, D. Hebert, P. Combis, and J.-L. Rullier, "Thermo-mechanical simulations of $\mathrm{CO}_{2}$ laserfused silica interactions," Journal of Applied Physics, vol. 119, p. 11, 2016.

[10] T. Doualle, L. Gallais, P. Cormont, T. Donval, L. Lamaignere, and J.-L. Rullier, "Effect of annealing on the laser induced damage of polished and $\mathrm{CO}_{2}$ laser-processed fused silica surfaces," Journal of Applied Physics, vol. 119, no. 21, pp. 3106-3111, 2016.

[11] D. L. Griscom, E. J. Friebele, and G. H. Sigel, "Observation and analysis of the primary 29Si hyperfine structure of the $\mathrm{E}^{\prime}$ center in non-crystalline $\mathrm{SiO}_{2}$," Solid State Communications, vol. 15, no. 3, pp. 479-483, 1974.

[12] A. F. Zatsepin, D. Y. Biryukov, and V. S. Kortov, "Analysis of OSEE spectra of irradiated dielectrics," Latvian Journal of Physics and Technical Sciences, vol. 6, p. 83, 2000.

[13] G. Pacchioni, L. Skuja, and D. L. Griscom, Defects in SiO2 and Related Dielectrics: Science and Technology, p. 73, Springer, Berlin, Germany, 2000.

[14] D. L. Griscom, "Optical properties and structure of defects in silica glass," Journal of the Ceramic Society of Japan, vol. 99, p. $899,1991$.

[15] L. Rebohle, J. von Borany, H. Fröb, T. Gebel, M. Helm, and W. Skorupa, "Ion beam synthesized nanoclusters for siliconbased light emission," Nuclear Instruments and Methods in Physics Research Section B: Beam Interactions with Materials and Atoms, vol. 188, no. 1-4, pp. 28-35, 2002.

[16] S. G. Demos, M. Staggs, and M. R. Kozlowski, "Investigation of processes leading to damage growth in optical materials for large-aperture lasers," Applied Optics, vol. 41, no. 18, pp. 3628-3633, 2002.

[17] M. A. Norton, L. W. Hrubesh, Z. Wu et al., "Chemical etch effects on laser-induced surface damage growth in fused silica," in Proceedings of SPIE-The International Society for Optical Engineering, Orlando, FL, USA, April 2001.

[18] L.-J. Zhang, C. Zhang, J. Chen et al., "Formation and control of bubbles during the mitigation of laser-induced damage on fused silica surface," Acta Physica Sinica Chinese Edition, vol. 67, no. 1, 2018.

[19] V. Švrček, H. Fujiwara, and M. Kondo, "Luminescent properties of doped freestanding silicon nanocrystals embedded in MEH-PPV," Solar Energy Materials \& Solar Cells, vol. 93, pp. 774-778, 2009.

[20] Y. Li, H. Yan, K. Yang et al., "Surface molecular structure defects and laser-induced damage threshold of fused silica during a manufacturing process," Scientific Reports, vol. 7, no. 1, p. 17870, 2017.

[21] A. Stesmans, M. Jivanescu, S. Godefroo, and M. Zacharias, "Paramagnetic point defects at $\mathrm{SiO}_{2}$ /nanocrystalline $\mathrm{Si}$ interfaces," Applied Physics Letters, vol. 93, no. 2, Article ID 023123, 2008. 